Document downloaded from:

http://hdl.handle.net/10251/55020

This paper must be cited as:

Torregrosa, AJ.; Hoyas, S.; Pérez Quiles, MJ.; Mompó Laborda, JM. (2013). Bifurcation Diversity in an Annular Pool Heated from Below: Prandtl and Biot Numbers Effects. Communications in Computational Physics. 13(2):428-441. doi:10.4208/cicp.090611.170212a.

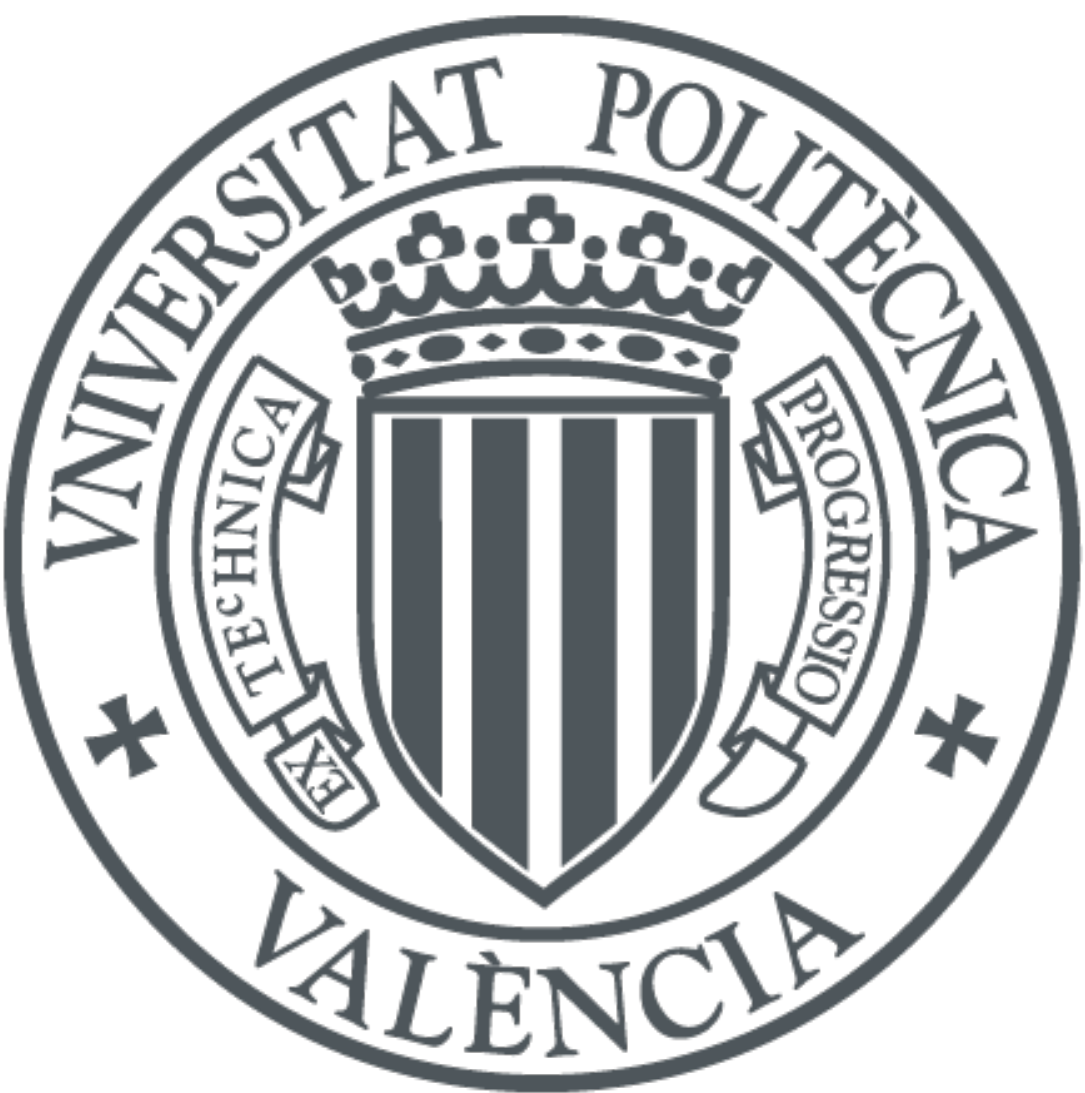

The final publication is available at

http://dx.doi.org/10.4208/cicp.090611.170212a

Copyright Global Science Press

Additional Information 


\title{
Bifurcation diversity in an annular pool heated from below: Prandtl and Biot numbers effects
}

\author{
A. J. Torregrosa ${ }^{1}$, S. Hoyas ${ }^{* 1}$, M. J. Pérez-Quiles ${ }^{2}$ and J. M. Mompó- \\ Laborda $^{1}$ \\ ${ }^{1}$ CMT-Motores Térmicos, Universitat Politècnica de València, Valencia 46022, Spain. \\ ${ }^{2}$ Instituto Universitario de Matemática Pura y Aplicada, Universitat Politécnica de \\ València, Valencia 46022, Spain.
}

\begin{abstract}
In this article the instabilities appearing in a liquid layer are studied numerically by means of the linear stability method. The fluid is confined in an annular pool and is heated from below with a linear decreasing temperature profile from the inner to the outer wall. The top surface is open to the atmosphere and both lateral walls are adiabatic. Using the Rayleigh number as the only control parameter, many kind of bifurcations appear at moderately low Prandtl numbers, and depending on the Biot number. Several regions on the Prandtl-Biot plane are identified, their boundaries being formed from competing solutions at codimension-two bifurcation points.
\end{abstract}

Key words: Thermocapillary convection, Prandtl number, Biot number, linear stability

\section{Introduction}

The problem of thermoconvective instabilities in fluid layers driven by a temperature gradient has become a classical subject in fluid mechanics [1], [28]. Two different effects are responsible for the onset of motion when the temperature difference becomes larger than a certain threshold: gravity and capillary forces. When both effects are taken into account the problem is called Bénard-Marangoni (BM) convection [1]. Classically, heat is applied uniformly from below [1] where the conductive solution becomes unstable for increasing temperature gradients. A more general set-up may be considered which includes thermoconvective instabilities by imposing a basic dynamic flow through nonzero horizontal temperature gradients, either in rectangular geometries $[3,6,10,13,15,17$, $21,22,28]$ or in cylindrical and annular geometries $[7,8,12,13,19]$. In particular, references $[8,14]$ and Garnier's PhD thesis [9] include a revision of the flow configuration found in

*Corresponding author. Email addresses: atorreg@mot.upv.es (A. Torregrosa), serhocal@mot.upv.es (S. Hoyas), jperezq@mat.upv.es (J. Pérez-Quiles), juamomla@mot.upv.es (J.M. Mompó-Laborda) 
this sort of problems. It is also worthy mentioning the experimental work of Schwabe et al [24], performed in low gravity conditions.

These studies are characterized by a set of dimensionless numbers:

1. Rayleigh number, $\mathrm{Ra}=g \alpha \Delta T d^{4} / \kappa v$ : Representative of the buoyancy effect.

2. Marangoni number, $\mathrm{Ma}=\gamma \Delta T d^{2} / \rho \kappa v$ : Accounts for the surface tension effects.

3. Prandtl number, $\operatorname{Pr}=v / \kappa$ : The ratio of momentum diffusivity (kinematic viscosity) to thermal diffusivity. In this article Pr values range from 1 to 20.

4. Bond number, $\mathrm{Bo}=\mathrm{Ra} / \mathrm{Ma}=\alpha \rho d^{2} / \gamma$ : Ratio of Rayleigh to Marangoni numbers, which is kept constant in this article.

5. Biot Number, Bi: Accounts for heat transmission between the fluid and the atmosphere. Values inside the range [0.2-1.5] are explored in this article.

6. Aspect ratio, $\Gamma=\delta / d$.

Here $\gamma$ stands for the rate of change of surface tension with temperature, $\kappa$ is the thermal diffusivity, $v$ is the kinematic viscosity of the liquid, $\alpha$ is the thermal expansion coefficient, $g$ is the gravitational acceleration, $\Delta T$ stands for a temperature increment, and $\delta$ and $d$ are characteristic lengths to be defined later. The reference values used are similar to those employed in [12], so that Bo $\sim 70$ and buoyancy effects are dominant. In this work, Prandtl, Biot and Rayleigh numbers were supposed to be independent parameters, and their effects on the solution of the problem was carefully studied.

In recent years Shi, Peng and several collaborators have studied numerically an annular geometry, [23,25-27], with a method similar to that used in [12]. The main differences between these works and the present article is that in those contributions the effects of the Biot number were not considered, and that the lateral walls of the annular pool were conductive. Other approximations to this sort of problems have been proposed which make use of tools coming from functional analysis, see [20] for details and references therein, but the presence of a tangential derivative in the Marangoni condition (see next section) makes this approximation almost impracticable.

Results on this problem were obtained in $[12,13]$ which evidenced the importance of heat-related parameters in the development of the instabilities. In [14] the authors found that very diverse bifurcations are controlled by the Biot number, and compared their solutions with the experimental results obtained by the group of Garnier [8]. The main interest of this paper is to generalized the results of these works, removing the infinite-Prandtl number approximation.

The paper is structured as follows. In the second section the formulation of the problem and the numerical method used to solve it are presented. Then, in the third section the results are presented and discussed. In the fourth section conclusions are presented and future works are proposed. 


\section{Formulation and Numerical Method}

The considered physical situation is sketched in figure 1. A horizontal fluid layer of depth $d$ ( $z$ coordinate) is contained in the space limited by two concentric cylinders of radii $a$ and $a+\delta$ ( $r$ coordinate). In all the following, the aspect ratio $\Gamma=\delta / d$ is set to 4 and $a=\delta$. The bottom plate is rigid and the top is open to the atmosphere, while the lateral walls are both adiabatic. A decreasing linear temperature profile from the inner to the outer cylinder was imposed on the bottom plate. The horizontal temperature gradient at the bottom had a value of $T_{G}=2 \mathrm{~K}$ and remained constant throughout the computations. The temperature used in the definition of the Rayleigh and Marangoni numbers was the mean temperature difference between the bottom plate and the atmosphere, $\Delta T$.

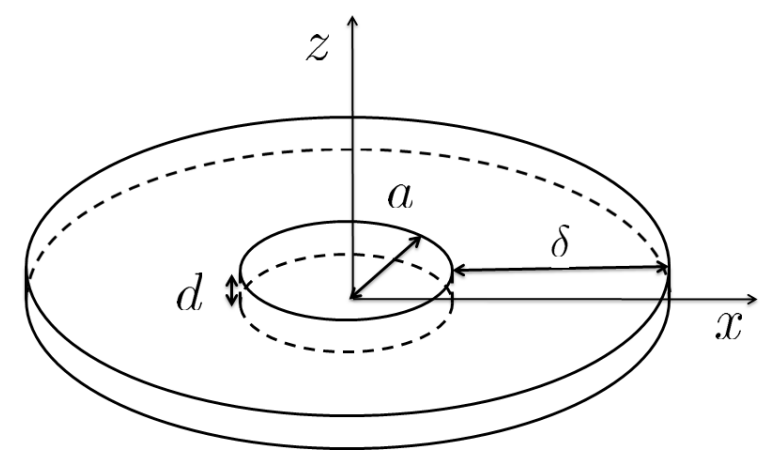

Figure 1: Sketch of the geometry. Lateral walls are considered adiabatic. The fluid is heated from below and the top surface is open to the atmosphere.

The system evolves according to the momentum and mass balance equations and to the energy conservation principle, non-dimensionalized as in $[5,12]$. As usual in this sort of problems, the Bousinesq approximation was used. In the equations governing the system $u_{r}, u_{\theta}$ and $u_{z}$ are the components of the velocity field $\mathbf{u}, \Theta$ is the temperature and $p$ is the pressure. The non-dimensionalized equations then become

$$
\begin{aligned}
\nabla \cdot \mathbf{u} & =0 \\
\partial_{t} \mathbf{u}+(u \cdot \nabla) \mathbf{u} & =\operatorname{Pr}\left(\nabla p+\nabla^{2} \mathbf{u}+\operatorname{Ra} \Theta \mathbf{e}_{z}\right), \\
\partial_{t} \Theta+\mathbf{u} \cdot \nabla \Theta & =\nabla^{2} \Theta .
\end{aligned}
$$

In these equations the operators and fields are expressed in cylindrical coordinates and $\mathbf{e}_{z}$ is the unit vector in the $z$ direction.

Boundary conditions are similar to those of references $[12,13]$ and are summarized in Table 1. Briefly, the velocity on the cylinders and the bottom plate correspond to rigid walls. In the top surface, the thermo-capillarity forces are modeled trough the Marangoni condition [12], whereas for the heat transmission the Biot condition is used. As is said 
previously, both lateral walls are considered adiabatic and a linear profile of temperature is imposed at the bottom.

\begin{tabular}{|l|l|l|}
\hline$z=0$ & $z=d$ & $r=a, a+\delta$ \\
\hline \hline$u_{r}=0$ & $\partial_{z} u_{r}+\mathrm{Ma}_{r} \Theta=0$ & $u_{r}=0$ \\
$u_{\phi}=0$ & $r \partial_{z} u_{\phi}+\mathrm{Ma}_{\phi} \Theta=0$ & $u_{\phi}=0$ \\
$u_{z}=0$ & $u_{z}=0$ & $u_{z}=0$ \\
$\Theta=\Delta T-\left(T_{G} / \delta\right) r$ & $\partial_{z} \Theta+\mathrm{Bi} \Theta=0$ & $\partial_{n} \Theta=0$ \\
\hline
\end{tabular}

Table 1: Boundary conditions.

\subsection{Basic state}

As soon as a temperature gradient is imposed, the fluid evolves until a stationary convective motion is reached, called basic state. Due to the symmetry of the problem and given that the flow is laminar, it is possible to consider a 2D axisymmetric approximation to this problem. In this case, any dependence on $\phi$ is neglected. Thus, the equations become

$$
\begin{aligned}
r^{-1} \partial_{r}\left(r u_{r}\right)+\partial_{z} u_{z} & =0, \\
\operatorname{Pr}^{-1}\left(u_{r} \partial_{r} u_{r}+u_{z} \partial_{z} u_{r}\right) & =-\partial_{r} p+\Delta_{c} u_{r}-\frac{u_{r}}{r^{2}} \\
\operatorname{Pr}^{-1}\left(u_{r} \partial_{r} u_{z}+u_{z} \partial_{z} u_{z}\right) & =-\partial_{z} p+\Delta_{c} u_{z}+\operatorname{Ra} \Theta, \\
u_{r} \partial_{r} \Theta+u_{z} \partial_{z} \Theta & =\Delta_{c} \Theta
\end{aligned}
$$

where $\Delta_{c}=r^{-1} \partial_{r}\left(r \partial_{r}\right)+\partial_{z}^{2}$ is the Laplacian operator in cylindrical coordinates. These equations are supplemented with the appropriate boundary conditions coming from Table 1.

In order to solve this problem a collocation method was used. The fluid magnitudes were expanded in a truncated series of orthonormal Chebyshev polynomials, as

$$
\begin{aligned}
& u_{r}(r, z) \simeq \sum_{n=0}^{N} \sum_{m=0}^{M} a_{n m} T_{n}(r) T_{m}(z), \\
& u_{z}(r, z) \simeq \sum_{n=0}^{N} \sum_{m=0}^{M} b_{n m} T_{n}(r) T_{m}(z), \\
& \Theta(r, z) \simeq \sum_{n=0}^{N} \sum_{m=0}^{M} c_{n m} T_{n}(r) T_{m}(z), \\
& p(r, z) \simeq \sum_{n=0}^{N} \sum_{m=0}^{M} d_{n m} T_{n}(r) T_{m}(z),
\end{aligned}
$$


where the unknowns are the polynomial coefficients $a_{n m}, b_{n m}, c_{n m}$, and $d_{n m}$. These polynomials were evaluated in the Chebyshev-Gauss-Lobatto (CGL) points [4],

$$
\begin{aligned}
& r_{i}=\cos \left(\pi \frac{i}{N}\right), j=0,1, \ldots, N, \\
& z_{i}=\cos \left(\pi \frac{i}{M}\right), j=0,1, \ldots, M,
\end{aligned}
$$

where $N$ and $M$ correspond to the order of the method. Non-linearities are solved by using a Newton-like iterative method, where the first approximation is either a slightly different basic state previously computed, or a solution of the system neglecting the nonlinearities. In both cases, the solution is exactly the same. This method works as follows:

Let $X^{i}=\left(u_{r}^{i}, u_{z}^{i}, \Theta^{i}, p^{i}\right)$ be the approximation at step $i$. The next step is defined as $X^{i+1}=X^{\varepsilon}+X^{i}$ being $X^{\varepsilon}$ much smaller than $X^{i}$. Imposing $X^{i+1}$ as a solution of (2.4-2.7), and neglecting non-linear terms including only $X^{\varepsilon}$ terms, a linear problem for $X^{\varepsilon}$ is obtained. This procedure is finished when the $l^{2}$ norm of each component of $X^{\varepsilon}$ is less than a prefixed error. Typically, a threshold of $10^{-9}$ was used, obtaining convergence in less than 20 iterations.

By using this technique, the problem of the spurious modes [2] arose, which was solved by using the method proposed in $[11,16]$, i.e., taking the normal projection of the momentum equations onto the boundary and replacing this condition at the top by the free divergence equation. As in [12], a test on the convergence of the method has been carried out and it will be described at the end of this section.

\subsection{Linear stability}

The linear stability analysis provides the critical Rayleigh number values and the shape of growing instabilities for fixed Prandtl and Biot numbers. Basic solutions are perturbed with fields depending on $r, \phi$ and $z$, in a full 3D analysis. Due to the periodicity in $\phi$, fluid magnitudes may be expanded in Fourier modes in $\phi$ as

$$
\begin{aligned}
u_{r}(r, \phi, z) & =u_{r}^{b}(r, z)+\bar{u}_{r}(r, z) e^{i k \phi+\lambda t,}, \\
u_{\phi}(r, \phi, z) & =u_{\phi}^{b}(r, z)+\bar{u}_{\phi}(r, z) e^{i k \phi+\lambda t}, \\
u_{z}(r, \phi, z) & =u_{z}^{b}(r, z)+\bar{u}_{z}(r, z) e^{i k \phi+\lambda t,}, \\
\Theta(r, \phi, z) & =\Theta^{b}(r, z)+\Theta(r, z) e^{i k \phi+\lambda t}, \\
p(r, \phi, z) & =p^{b}(r, z)+p(r, z) e^{i k \phi+\lambda t} .
\end{aligned}
$$

where $k \geq 0$ is the wavenumber and superscript $b$ denotes the basic state. The eigenvalue $\lambda$ characterizes the instability: when its real part is negative the basic state is stable whereas if it is positive the basic solution is unstable. In this case, the imaginary part of $\lambda$ can be either zero -the bifurcation is stationary- or non zero -the bifurcation is oscillatory. In the first case, the stationary longitudinal rolls solution (SR) is observed. In the second case, 

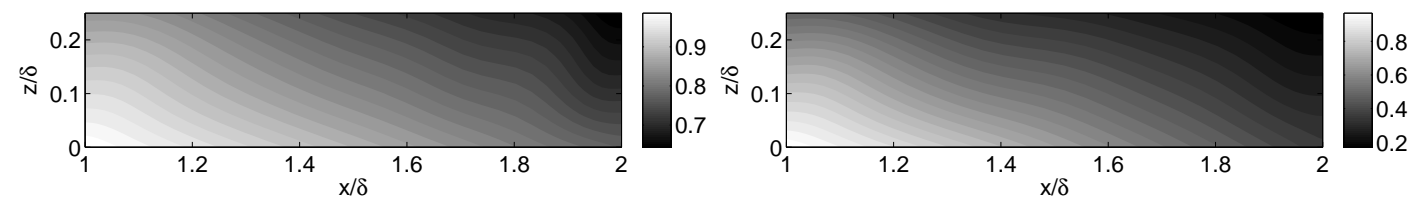

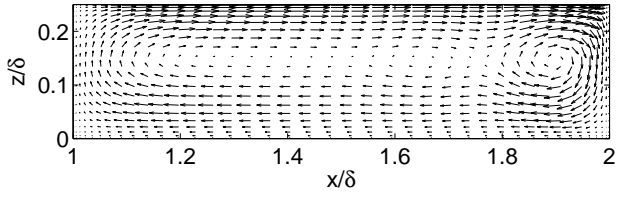

(a) $\operatorname{Pr}=1, \mathrm{Bi}=0.2, R a_{c}=5775$
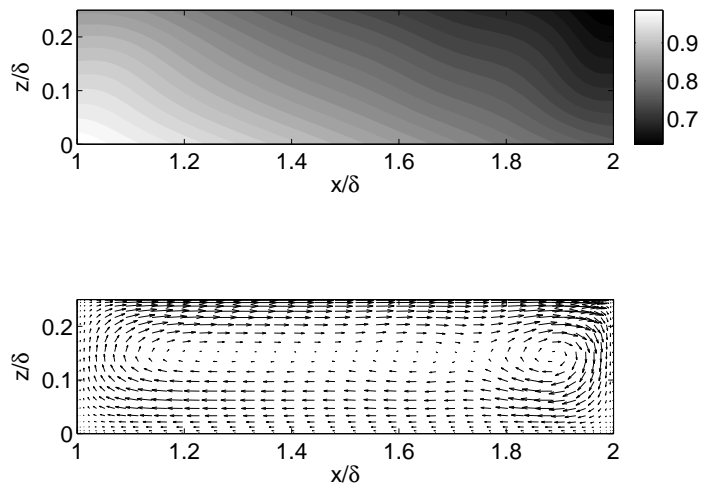

(c) $\operatorname{Pr}=10, \mathrm{Bi}=0.2, R a_{c}=4546$

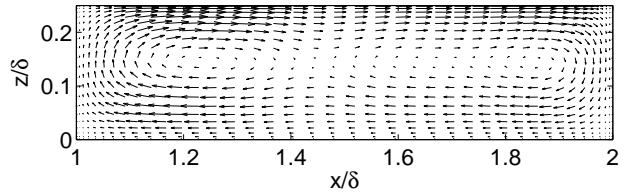

(b) $\operatorname{Pr}=1, \mathrm{Bi}=1.2, R a_{c}=1956$
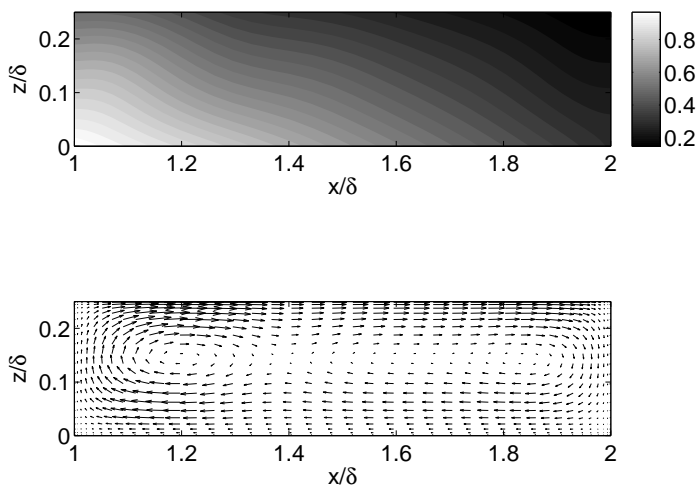

(d) $\operatorname{Pr}=10, \mathrm{Bi}=1.2, R a_{c}=2151$

Figure 2: Velocity diagram and temperature distribution of the basic state in $x-z$ plane for several Prandtl, Biot and Rayleigh numbers. In all cases, Rayleigh number is the critical one. Temperature has been normalized by the maximum value of $\Theta$ in each case. $\mathrm{Ra}$

the system exhibits hydrothermal waves (HW). In the range of Biot and Prandtl numbers studied it has always been possible to find a Rayleigh number such that the basic state is stable. For higher values of the Rayleigh number the basic state becomes unstable, and the shape and class of the growing solution depends only on the Biot and the Prandtl numbers. In all cases, as Bond number is constant, the Marangoni number is such that $\mathrm{Ma}=\mathrm{Ra} / \mathrm{Bo}$

In order to compute the eigenvalues of the problem, equations (2.12-2.16) are introduced into equations, (2.1-2.3), and after neglecting the nonlinear terms, and recalling that the basic state is a solution of the problem (2.4-2.7), the non-dimensionalized equa- 


\begin{tabular}{|l|l|l|}
\hline$z=0$ & $z=d$ & $r=a, a+\delta$ \\
\hline \hline$u_{r}=0$ & $\partial_{z} u_{r}+\mathrm{Ma}_{r} \Theta=0$ & $u_{r}=0$ \\
$u_{\phi}=0$ & $\partial_{z} u_{\phi}+i r^{-1} k \mathrm{Ma} \Theta=0$ & $u_{\phi}=0$ \\
$u_{z}=0$ & $u_{z}=0$ & $u_{z}=0$ \\
$\Theta=0$ & $\partial_{z} \Theta+\mathrm{Bi} \Theta=0$ & $\partial_{n} \Theta=0$ \\
\hline
\end{tabular}

Table 2: Boundary conditions for the linear stability problem

tions of the basic state become

$$
\begin{aligned}
\partial_{r}\left(r \bar{u}_{r}\right)+i k \bar{u}_{\phi}+\partial_{z} r \bar{u}_{z} & =0 \\
\sigma^{-1}\left(\lambda \bar{u}_{r}+u_{r}^{b} \partial_{r} \bar{u}_{r}+\bar{u}_{r} \partial_{r} u_{r}^{b}+u_{z}^{b} \partial_{r} \bar{u}_{r}+\bar{u}_{z} \partial_{r} u_{r}^{b}\right) & = \\
-\partial_{r} \bar{p}+\Delta_{k} \bar{u}_{r}-r^{-2}\left(\bar{u}_{r}+2 i k \bar{u}_{\phi}\right) & \\
\sigma^{-1}\left(\lambda \bar{u}_{\phi}+u_{r}^{b} \partial_{r} \bar{u}_{\phi}+u_{z}^{b} \partial_{z} \bar{u}_{\phi}+r^{-1} u_{r}^{b} \bar{u}_{\phi}\right) & = \\
-\frac{1}{r} i k \bar{p} & +\Delta_{k} \bar{u}_{\phi}+r^{-2}\left(2 i k \bar{u}_{r}-u_{\phi}\right) \\
\sigma^{-1}\left(\lambda \bar{u}_{z}+u_{r}^{b} \partial_{r} \bar{u}_{z}+\bar{u}_{z} \partial_{r} u_{r}^{b}+u_{z}^{b} \partial_{z} \bar{u}_{z}+\bar{u}_{z} \partial_{z} u_{z}^{b}\right) & =\partial_{z} \bar{p}+\Delta_{k} \bar{u}_{z}+\operatorname{Ra} \bar{\Theta}_{z} \\
\lambda \Theta+u_{r} A \partial_{r} \Theta \Theta^{b}+u_{r}^{b} A \partial_{r} \Theta+2 u_{z}^{b} \partial_{z} \Theta+2 u_{z} \partial_{z} \Theta & =\Delta_{k} \Theta
\end{aligned}
$$

where $\Delta_{k}=\partial_{r}^{2}+r^{-1} \partial_{r}-k^{2} r^{-2}+\partial_{z}^{2}$. Boundary conditions are given in Table 2 .

This is a generalized eigenvalue problem, of the form

$$
A X=\lambda B X
$$

where $X=\left(\bar{a}_{n m}, \bar{b}_{n m}, \bar{c}_{n m}, \bar{d}_{n m}, \bar{e}_{n m}\right)$ is the vector containing the polynomial coefficients of the perturbations and $\bar{e}_{n m}$ are the coefficients of the $u_{\phi}$ expansion. $A$ and $B$ are full complex matrices, of dimension $5(N+1)(M+1)$. It is clear from the equations and boundary conditions that $\lambda$, which is a vector of dimension $5(N+1)(M+1)$, is a function of $\mathrm{Ra}, \mathrm{Bi}$, Pr, and $k$.

In previous works, in which the $\operatorname{Pr}=\infty$ approximation was used, the computation time for the eigenvalues was two orders of magnitude larger than that required for the basic state, which strongly limits the amount of cases that can be studied. However, a new algorithm developed by Navarro et al [18], and specially designed for the matrix structure of these sorts of problems, accelerated notably this computation, with a total time reduction of nearly $70 \%$. The numerical approach of this article (generalized Arnoldi method) uses the idea of preconditioning the eigenvalue problem with a modified Cayley transformation before applying the Arnoldi method, computing only the eigenvalues with greater real part. All the algorithms described above, including this last one, were implemented in Fortran 90 using standard BLAS and LAPACK routines. 


\subsection{Critical eigenvalue and convergence}

As it has been mentioned above, for any Biot-Prandtl pair lying in the intervals studied, it was always possible to find a Rayleigh number such that the eigenvalue with the maximum real part, $\mu=\max (\operatorname{Re}(\lambda))$ is always lower than 0 , i.e., the basic state is stable. As the Rayleigh number is increased, and keeping Pr and Bi constants, a critical Rayleigh number exists, defined by the condition

$$
\min _{k \in I} \mu\left(\mathrm{Ra}_{c}^{k}, k\right)=0
$$

where $I$ is the wavenumber range studied. The search for this critical eigenvalue was accelerated through an extrapolation iterative method. This algorithm usually obtains $\mathrm{Ra}_{c}^{k} \leq 10^{-6}$ in less than 5 iterations. In Figure 3, the marginal stability diagram for two different cases is shown. In Figure 3a the value of $\mu$ is shown for several Rayleigh numbers and for $\operatorname{Pr}=20$ and $\mathrm{Bi}=0.7$. Two branches of competing solutions can be observed, one stationary and the other oscillatory. In this Figure, complex eigenvalues are denoted by using closed symbols, whereas open ones denote real eigenvalues. In this case, the right branch reaches zero for $\mathrm{Ra}_{c}^{k}=2489$ and $k_{c}=17$, the corresponding bifurcation being stationary. In this case this eigenvalue codimension is one. On the contrary, in Figure $3 \mathrm{~b}$, it is found that, for the same $\operatorname{Ra}_{c}=2608, \mu\left(\operatorname{Ra}_{c}, 13\right)=\mu\left(\operatorname{Ra}_{c}, 17\right)=0$. This is an example of codimension-two bifurcations. It was found that curves formed by (Pr,Bi) pairs where a codimension-two bifurcation takes place, divide the Biot-Prandtl plane in several regions (see Figure 5). In each of these three regions the growing perturbation are completely different in shape, as will be discussed in next section.

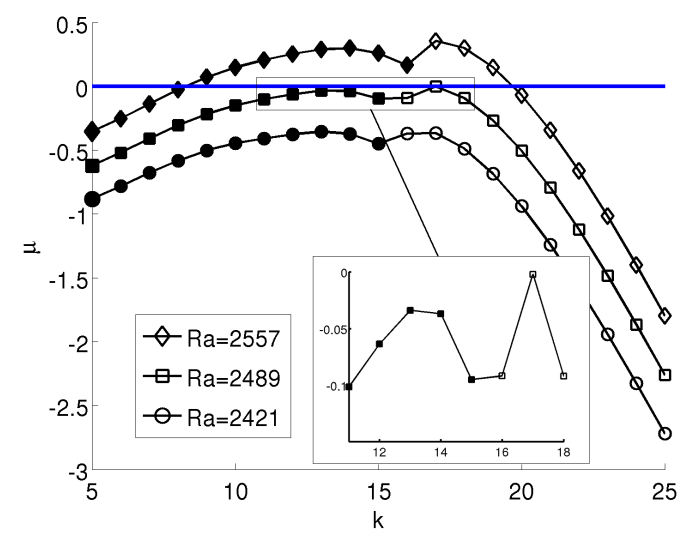

(a) $\operatorname{Pr}=20, B=0.7$

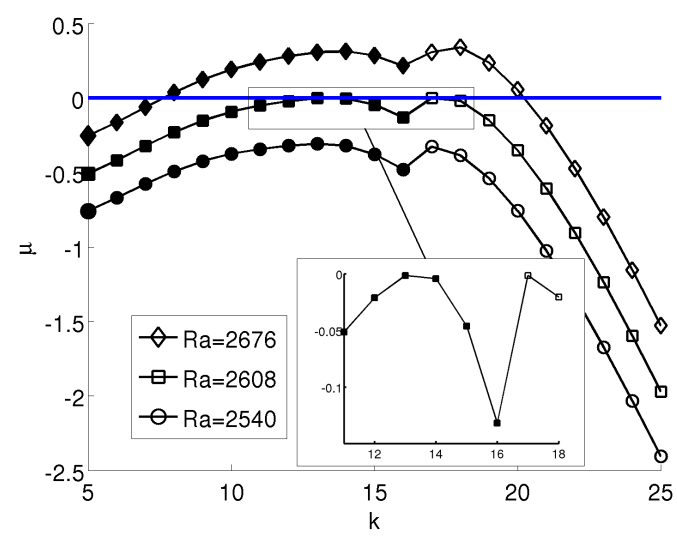

(b) $\operatorname{Pr}=10, \mathrm{~B}=0.655$

Figure 3: Marginal stability diagrams. Codimension-1 (3a) and 2 (3b) bifurcations are shown. Closed symbols denote complex eigenvalues, whereas open symbols stand for real ones. 


\begin{tabular}{|c|c|c|c|c|c|c|c|}
\hline $\operatorname{Pr}$ & $21 \times 13$ & $33 \times 13$ & $21 \times 17$ & $33 \times 17$ & $21 \times 21$ & $33 \times 21$ & $k_{c}$ \\
\hline 40 & 1921.79 & 1921,56 & 1921,79 & 1921.56 & 1921.79 & 1921.56 & 14 \\
\hline 30 & 1927.65 & 1927.38 & 1927.65 & 1927.38 & 1927.65 & 1927.38 & 14 \\
\hline 20 & 1934.76 & 1934.69 & 1934.76 & 1934.69 & 1934.76 & 1934.69 & 15 \\
\hline 10 & 1956.57 & 1956.51 & 1956.57 & 1956.51 & 1956.57 & 1956.51 & 15 \\
\hline 5 & 1992.46 & 1992.08 & 1992.46 & 1992.08 & 1992.46 & 1992.08 & $13 i$ \\
\hline 1 & 2151.11 & 2151.62 & 2151.11 & 2151.62 & 2151.05 & 2151.52 & 0 \\
\hline
\end{tabular}

Table 3: Critical Rayleigh number for several expansions of $N$ and $M$. Six digits of precision are reached for $M=13$ in all cases

The convergence of the method was estimated as in [12], both in $r$ and $z$. In Table 3 the values of $\mathrm{Ra}_{c}$ for several $(N, M)$ expansion pairs are shown. In every case there is coincidence in the critical wavenumber for each Prandtl number. As it is clear from the table, convergence in $z$ is obtained for $M=13$. Results for convergence in $N$ for some Prandtl numbers are plotted in Figure 4. In all the cases (not shown), the critical wavenumber remains constant for each Prandtl number. In this figure the relative error $\varepsilon$ referred to $N=39$,

$$
\varepsilon_{n}=\frac{\mathrm{Ra}_{c}^{n}-\mathrm{Ra}_{c}^{39}}{\mathrm{Ra}_{c}^{39}}
$$

has been plotted. It is always lower than $10^{-4}$ for $n$ greater than 21 . As the shape and type of the growing perturbation was the main interest of this work, all the simulations were performed with $N=21$ and $M=13$, which was sufficient for this purpose.

\section{Discussion}

\subsection{Basic state}

In the range of Prandtl numbers studied, the shape of the basic state at the critical Rayleigh number depends mostly on the Biot number, see Figure 2. This agrees with the results shown in [14], where the authors found that for $\operatorname{Pr}=\infty$ the basic state shape was characterized by the Biot number, the aspect ratio and the horizontal temperature gradient. This is not unexpected, taking into account that the latter are constant. However, it is remarkable that the Prandtl number does not affect the basic state. Probably this is due to the fact that $\operatorname{Pr} \geq 1$ was considered in this work. The basic state is formed by two co-rotating rolls perpendicular to the temperature gradient. This result is similar to that obtained in the experiments reported in $[3,6]$, where co-rotating rolls perpendicular to the gradient were also found, and is also in good agreement with [8].

It is also worthy mentioning that in all the cases simulated the temperature gradient always point from right to left. Evidence of vertical inversions of the temperature gra- 


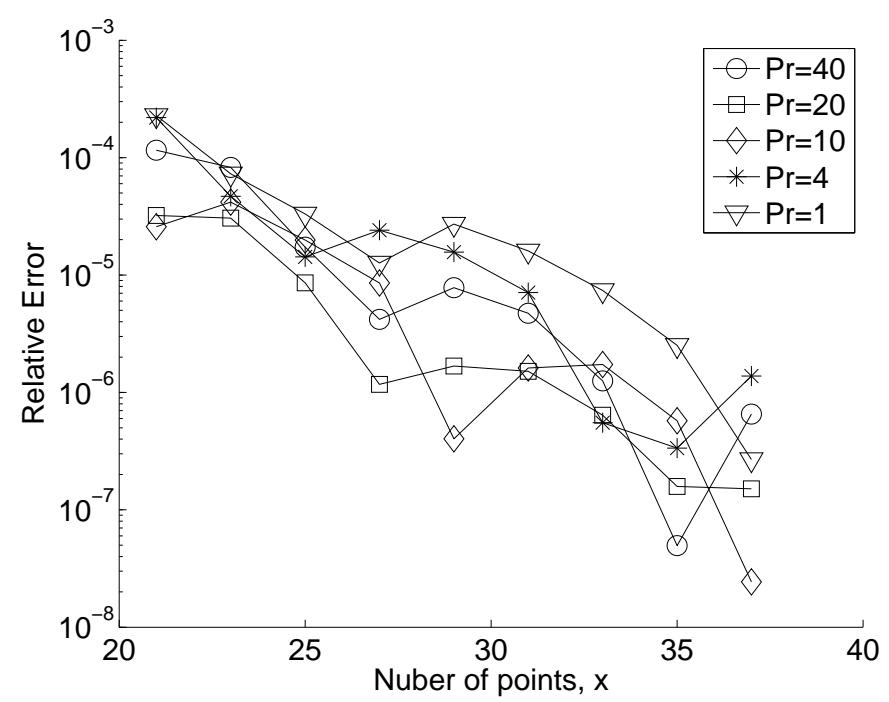

Figure 4: Relative error in the computation of the critical Rayleigh number for several Prandtl numbers and $\mathrm{Bi}=1.2$. Error for $n=35,37$ is in the range of the iterative method used to find $\mu$

dient "return flow" are not observed, contrarily to what it was reported at [14]. This is probably because return flow is an aspect-ratio related effect.

\subsection{Linear stability}

Depending on the shape of the growing bifurcation, there exist three regions in the plane $\mathrm{Pr}-\mathrm{Bi}$, referred to as I, II and III in Figure 5. The curves delimiting the regions are formed by codimension-two points as those plotted in $3 \mathrm{~b}$. Representative isotherms in the $r-z$ top plane of each perturbation zone are also shown. In Table 4, a summary of the main characteristics of each zone is given.

\begin{tabular}{|c|c|c|c|c|}
\hline Zone & Type & Wavenumber & Biot range & Prandtl range \\
\hline I & Stationary & 0 & $0.2-1.2$ & $18-1.5$ \\
\hline II & Oscillatory & $8-14$ & $\left\{\begin{array}{lll}0.2-1.2 & \text { if } & \operatorname{Pr}<6 \\
0.2-0.6 & \text { if } & \operatorname{Pr} \geq 6\end{array}\right.$ & $1.5-\infty$ \\
\hline III & Stationary & $\geq 16$ & $0.6-1.5$ & $5.7-\infty$ \\
\hline
\end{tabular}

Table 4: Main characteristics of the three regions found.

The dependence of the critical Rayleigh number on the Biot and Prandtl numbers is depicted in Figures $6 a$ and $6 b$, respectively. It is clear from these plots that if either 


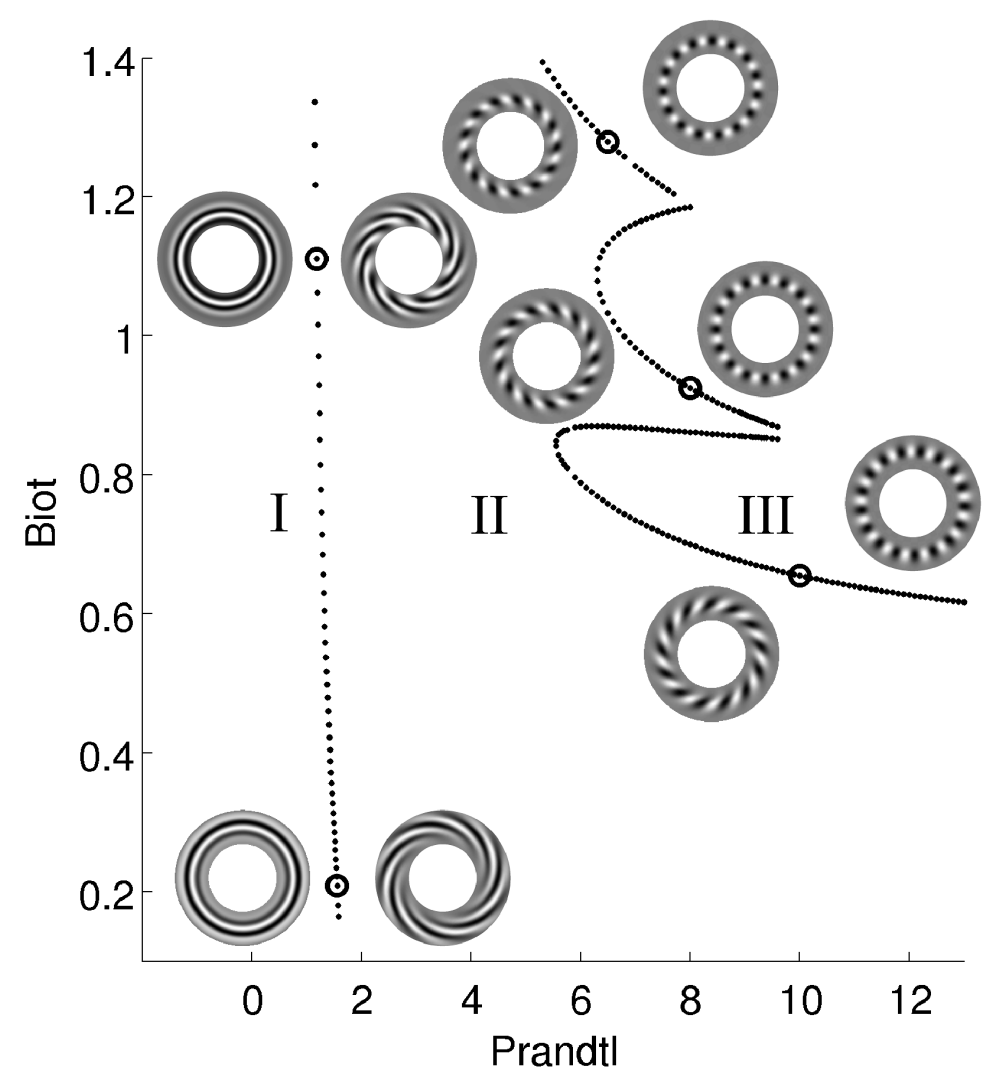

Figure 5: Prandtl-Biot plane stability diagram showing regions I, II and III. Representative top $r-\phi$ plane isotherms corresponding to marked points are shown

the Prandtl or the Biot number decreases, then $\mathrm{Ra}_{c}$ increases. In the case of the Biot number, the system stored more heat as it decreased, so that the Rayleigh number had to increase. The Prandtl number effects can also be easily understood if one thinks in terms of decreasing viscosity. Figure 6a also shows the transition between the different regions, due to the regime change of the growing solution. It is also clear, however, that there was almost no change in the profile of the curves, until Pr is close to 1.

The three-dimensional shape of the perturbations is quite different. Representative 3D structures of these perturbations can be seen in Figure 7. The growing solution of Region I presents several stationary longitudinal counter-rotating rolls, similar to those found by Garnier [8]. In this region, the influence of the Biot number on the shape is not quite evident, as can be seen in Figure 5. The boundary between Regions I and II seems to be strongly related to the Prandtl number as in the experiments of [28]. Dependencies on the Biot number are more evident in regions II and III. In Region II, HW are found for $\operatorname{Pr}<5$ for all Biot numbers. The shape of these HW depend on both the Prandtl 


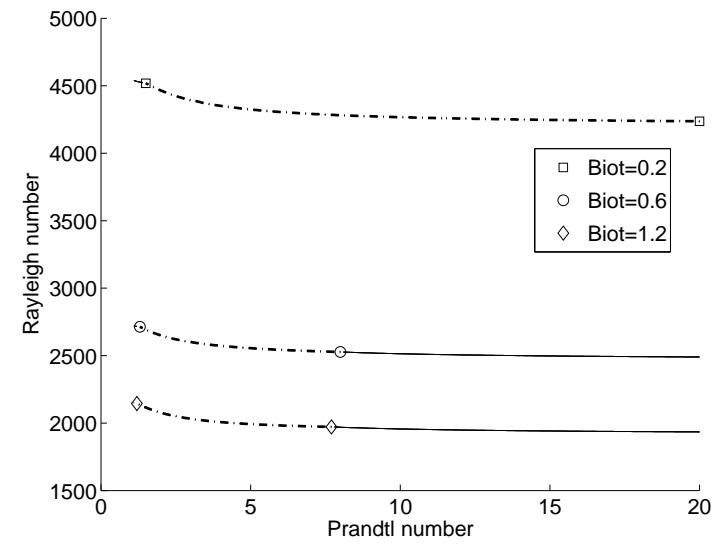

(a) Prandtl versus Rayleigh

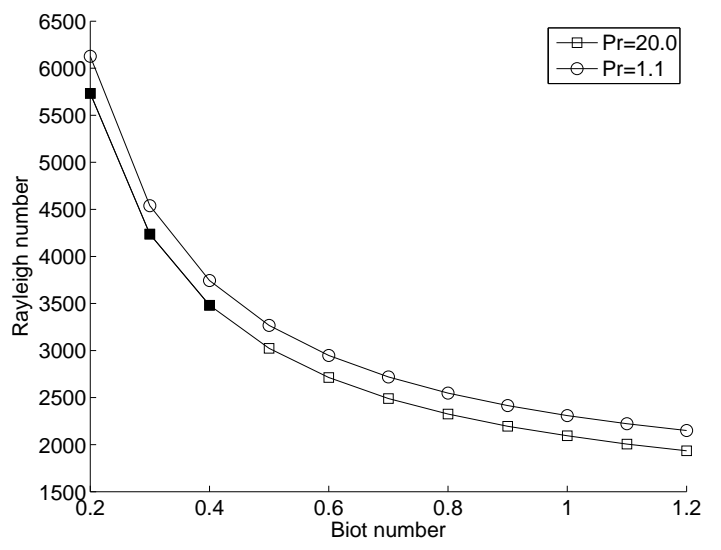

(b) Biot versusRayleigh

Figure 6: Critical Rayleigh number as a function of Prandtl number, figure 6a and Biot number $6 b$

and the Biot numbers. As the Biot number increases, the HW are shortened and their leftmost parts are closer to the hottest part (i.e. the inner cylinder) of the annulus. Prandtl number effects are essentially the same in this region. However, for Prandtl numbers ranging roughly from 5 to 10, the Biot number is critical in order to determine the type of the growing solution. For $\operatorname{Pr}>10$, the main parameter controlling the shape of the perturbation is the Biot number. The rightmost branch of the curve forming the boundary between regions II and III continues until $\mathrm{Pr}=100$ (not shown) for an almost constant value of $\mathrm{Bi}=0.47$. This agrees with the results of [14]. Lastly, Region III presents a SR solution, not showing any clear influence of the Prandtl number on the structure of the growing perturbation when $\operatorname{Pr} \geq 10$.

\section{Conclusions}

In this work the instabilities appearing in a cylindrical annulus heated from below were analyzed by means of the linear stability method. This thermo- and fluid-dynamic problem is governed by mass, momentum and energy conservation equations in primitive variables, where the Bousinesq approximation has been used. Basic state and linear stability equations were solved by using spectral methods. All the parameters and dimensionless numbers were fixed but three: the Rayleigh, Prandtl and Biot numbers

The main conclusion of this article is that for $\operatorname{Pr}<2$, approximately, the main parameter controlling the shape of the growing bifurcation is the Prandtl number. For Pr between 2 and 10, the Biot and Prandtl numbers are both important and for $\operatorname{Pr}>10$, the Bi value defines the shape of the growing bifurcation.

As a future work, the situation when Pr is below 1 is not clear, and the eventual exis- 


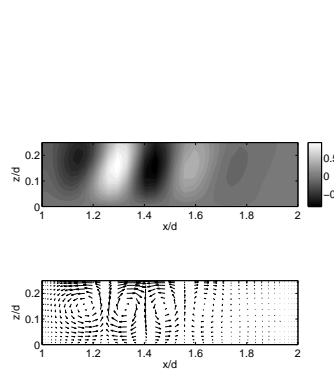

(a) $\operatorname{Pr}=1.18, \mathrm{Bi}=1.1$

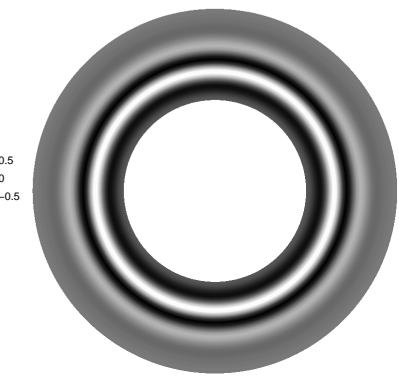

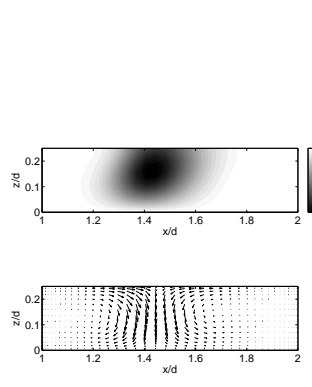

(c) $\operatorname{Pr}=8, \mathrm{Bi}=0.93$

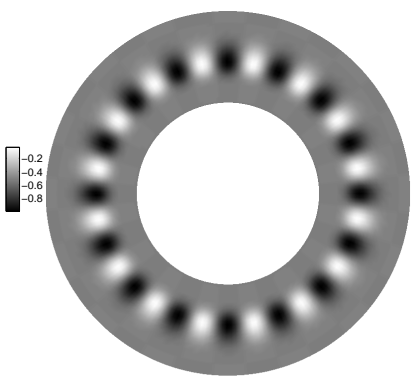

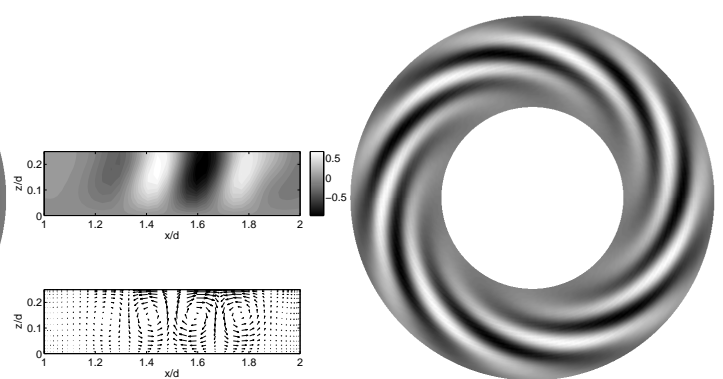

(b) $\operatorname{Pr}=1.55, \mathrm{Bi}=0.2$

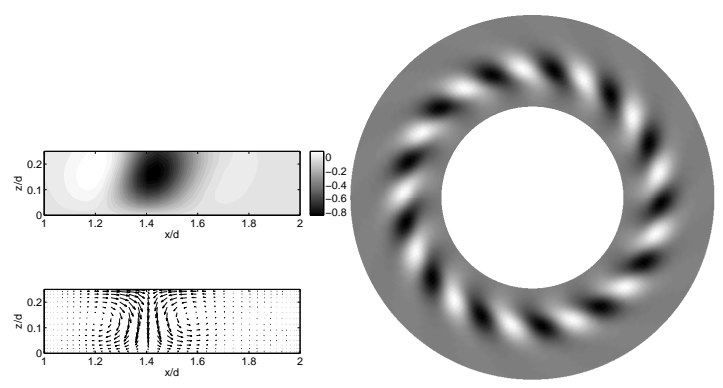

(d) $\operatorname{Pr}=8, \mathrm{Bi}=0.93$

Figure 7: Velocity diagram, temperature isotherms in $x-z$ plane and top view of the growing perturbation for several Prandtl and Biot numbers. Temperature has been normalized by the maximum value of $\Theta$ in each case.

tence of any new regions in the $\mathrm{Bi}-\mathrm{Pr}$ plane has not been studied. As it is necessary to increase the order of the method, this is a costly initiative. It would also be very interesting to verify the eventual influence of changes in the aspect ratio $\Gamma$, and try to replicate the results of [8]. 


\section{Aknowledgments}

The code developed for this article was finished during a visit of S. Hoyas to the Lehrstuhl für Strömungsmechanik of the University of Erlangen-Nürnberg, funded by the Government of Valencia and the Universitat Politècnica de València. S. Hoyas would like to thank to Prof. Delgado and Dr. Ausmeier for their support and kindness. Last, but not least, S. Hoyas is, and always will be, in debt with his PhD advisors, Prof. Herrero, Prof. Mancho and Prof. Pardo.

\section{References}

[1] H. Bénard Rev. Gén. Sci. Pures Appl. 11, 1261 (1900).

[2] C. Bernardi and Y. Maday. Approximations spectrales de problemes aux limites elliptiques. Springer-Verlag, Paris, 1992.

[3] J. Burguete, N. Mokolobwiez, F. Daviaud, N. Garnier and A. Chiffaudel, Phys. Fluids 13, 2773 (2001).

[4] C. Canuto. M. Y. Hussaini, A. Quarteroni and T. A. Zang. Spectral Methods in Fluid Dynamics. Springer-Verlag, Berlin, 1988.

[5] S. Chandrasekhar. Hydrodynamic and Hydromagnetic Stability. Dover Publications, New York, 1981.

[6] F. Daviaud and J.M. Vince, Phys. Rev. E 48, 4432 (1993).

[7] A.B. Ezersky, A. Garcimartín, J. Burguete, H.L. Mancini and C. Pérez-García, Phys. Rev. E 47, 1126 (1993).

[8] N. Garnier and A. Chiffaudel. Eur. Phys. J. B 19, 87 (2001).

[9] N. Ganier. PhD thesis, http:/ / nicolasgarnier.free.fr/these-garnier.pdf (2002)

[10] H. Herrero and A. M. Mancho Phys. Rev E 57, 7336 (1998).

[11] H. Herrero, S. Hoyas A. Donoso, A.M. Mancho, J.M. Chacon, R.F. Portugués and B. Yeste. J. Scientific Computing 18, (2), 315-328, 2003.

[12] S. Hoyas, H. Herrero and A.M. Mancho, J. Phys. A: Math and Gen. 35, 4067 (2002).

[13] S. Hoyas, H. Herrero and A.M. Mancho, Phys. Rev. E, 66, 057301 (2002).

[14] S. Hoyas, H. Herrero, A.M. Mancho, N. Garnier and A. Chiffaudel, Phys. Fluids 17, 054104 (2005)

[15] A. M. Mancho, H. Herrero and J. Burguete Phys. Rev E 56, 2916 (1997).

[16] A.M. Mancho and H. Herrero, Phys. Fluids 12, 1044 (2000).

[17] J. F. Mercier and C. Normand, Phys. Fluids 8, 1433 (1996).

[18] MC Navarro, H Herrero, A.M. Manch, A. Wathen, Commun. Comput. Phys. 3, 2, 308 (2008)

[19] MC Navarro, H Herrero, S Hoyas, Commun. Comput. Phys. 5, 2-4, 649-666 (2009)

[20] R. Pardo, H. Herrero, S. Hoyas, J. Math Anal Appl 376, 1, 231-246 (2011)

[21] M.A. Pelacho and J. Burguete, Phys. Rev. E 59, 835 (1999).

[22] R.J. Riley and G.P. Neitzel, J. Fluid Mech 359, 143 (1998).

[23] Peng L, Li YR, Shi WY and Imaishi N Int J. Heat Mass Tran 50,5-6, 872-880, (2007)

[24] D Schwabe,A Zebib and BC Sim J. Fluid Mech 491, 239-258, (2003)

[25] Shi WY, Ermakov MK, Li YR, Peng L and Imaishi N. Microgravity Sci. Tec. 21, 289-297, (2009)

[26] Shi Wy, Peng L, J. Eng. Thermophys-rus 21,2, Pages 250-254, (2011)

[27] Shi WY, Liu X, Li GY, Li YR, Peng L, Ermakov MK and Imaishi N J. Supercond Nov. Magn. 23,6, 1185-1188, (2010) 
[28] M. K. Smith and S. H. Davis J. Fluid Mech. 132, 119 (1983). 